\title{
La gestion automatisée d'aménagements fluviaux à buts multiples L'exemple du Rhône
}

\author{
Computerized management of fluvial \\ plants with multiple aims \\ The Rhône example
}

N. Bordiec

A. Frézet

Compagnie nationale du Rhône

2, rue André Bonin, 69316 Lyon Cedex 04

Les aménagements hydrauliques à buts multiples : production d'énergie électrique, navigation commerciale à grand gabarit, protection contre les crues, posent à leurs exploitants des problèmes de différents ordres liés aux types d'exploitation et de gestion envisagés.

Dans le cas du Rhône (18 aménagements en série sur $530 \mathrm{~km}$ ), le nombre et la variété des contraintes (aménagements en cascade, crues, diverses formes de l'exploitation des plans d'eau) ont conduit la Compagnie nationale du Rhône (CNR) à adopter une conduite automatique par ordinateurs, qui est décrite au cours de l'article.

Hydraulic plants with multiple aims : power generation, large scale merchant service, protection against floods pose to their operating companies various types of problems linked to the envisaged types of exploitation and management.

In the case of the Rhone (18 plants in series over $530 \mathrm{~km})$, the number and the diversity of the constraints, such as developments involving waterfalls, floods, various forms of water operation, have led the Compagnie Nationale du Rhône (CNR) to adopt automatic control using computers which is described in the article.

\section{Préambule}

Les aménagements hydrauliques à buts multiples : production d'énergie électrique, navigation commerciale à grand gabarit, protection contre les crues, posent à leurs exploitants des problèmes de différents ordres liés aux types d'exploitation et de gestion envisagés.

Dans le cas du Rhône, sur lequel a été créée une chaine d'aménagements (figure 1), 18 en série sur une distance de $530 \mathrm{~km}$, de basse chute (à l'exception d'un seul, celui de tête - Génissiat - doté d'un réservoir), la conduite et l'optimisation du fonctionnement présentent quelques difficultés du fait des problèmes et contraintes parfois contradictoires rencontrés et des diverses formes d'exploitation envisagées. On peut citer les suivantes: a) Aménagements en cascade : tout événement dans un aménagement quelconque a des répercussions immédiates ou à court terme sur les aménagements situés à l'aval.

b) Possibilité d'accumulation très limitée dans chaque aménagement, mais cependant obligation d'une tenue stricte des niveaux de consigne, aussi bien aux débits usuels qu'aux débits de crue pour éviter tout débordement sur les endiguements et toute gène à la navigation.

c) Crues nombreuses et importantes du fleuve et de ses affluents, dont les eaux doivent être contenues dans les limites du lit majeur du fleuve ou des endiguements et qui ne doivent être ni accélérées, ni amplifiées par les aménagements hydrauliques, les deux rives du fleuve étant occupées par une population dense exerçant des activités industrielles et agricoles. 


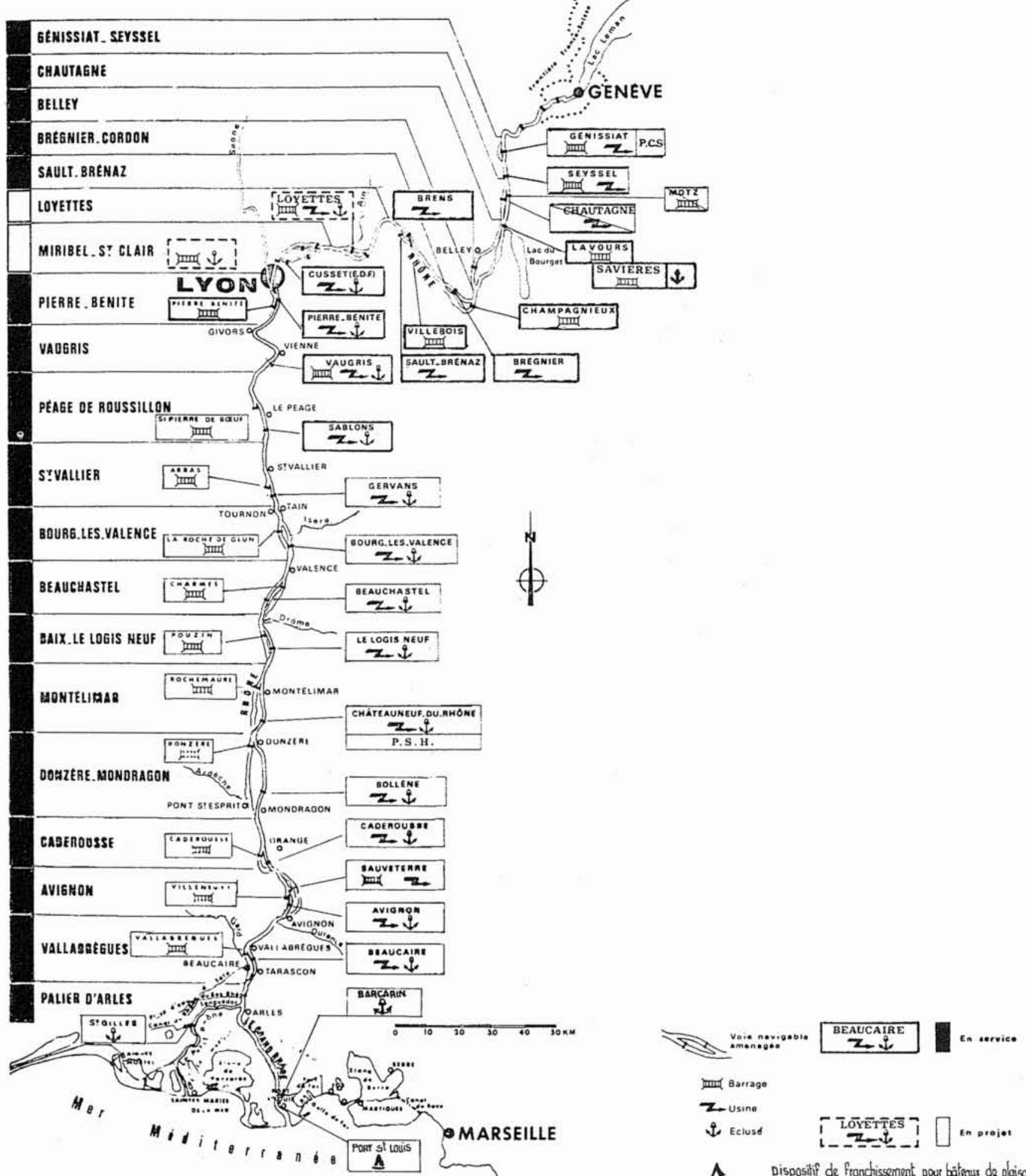

A Disposilifi de franchissement pour bathers de plaisance 
d) Exploitation des plans d'eau sous des formes diverses afin d'obtenir les meilleures performances possibles dans les productions hydroélectriques :

- éclusée individuelle pour obtenir le meilleur placement de l'énergie suivant la variation quotidienne de la demande : une centrale fonctionne sur programme de débit, alors que celles de l'aval sont conduites au fil de l'eau, donc en réglage de niveau, malgré les perturbations venant de l'amont;

- éclusées cumulatives : un certain nombre d'aménagements fonctionnent en programme de débit, en synchronisme ou non, ce qui entraine des grandes variations de débit qui induisent des variations de niveau qu'il faut cependant contenir à l'intérieur de limites données;

- contribution à des "pointes " brusques de puissance pour le sauvetage du réseau électrique qui conduit un certain nombre d'aménagements successifs à faire des variations de débit très rapides, importantes et cumulatives (saturation des groupes hydroélectriques) qui entrainent des modifications importantes de la pente dés lignes d'eau avec risque d'oscillations des plans d'eau;

- contribution au téléréglage fréquence-puissance du réseau, lequel par les variations de débit aléatoires qui découlent de ce type de fonctionnement, constitue une souce de "bruits parasites " gênant pour le réglage des niveaux.

Toutes ces sujétions, difficilement maitrisables en exploitation manuelle, ont conduit la Compagnie nationale du Rhône (C.N.R.) a adopter une conduite automatique (1) par ordinateurs, non seulement des ouvrages réglant le débit mais aussi des plans d'eau sur le cours entier du fleuve français, avec l'objectif d'affiner et d'optimiser le fonctionnement de chacun des aménagements, mais aussi celui de l'ensemble de la chaine, et enfin celui d'assurer une sécurité vis-à-vis des ouvrages et des riverains au moins égale à celle assurée par du personnel permanent de surveillance.

Il a fallu ainsi, après étude des lignes d'eau ainsi que des consignes d'exploitation imposées par le Cahier des charges des aménagements, concevoir et construire des modèles mathématiques qui soient les plus représentatifs possible des aménagements, étudier les lois de réglage en dynamique, concevoir les algorithmes de régulation et les intégrer dans un système informatisé temps réel.

La C.N.R. a dû s'imprégner des conditions et modalités pratiques de la conduite afin de les prendre en compte dans le système "automatisé " - imaginer la conduite sous l'angle primordial de la sécurité, prendre en considération les défauts de matériels et les disfonctionnements et étudier la conduite en situation dégradée, les moyens et conditions de repli puis d'appel du personnel astreint à domicile.

\section{Généralités sur l’aménagement du Rhône}

L'aménagement du Rhône s'étend sur une longueur de $530 \mathrm{~km}$, de la frontière franco-suisse à la mer.

Le Bas-Rhône, de Lyon à Arles entièrement aménagé depuis 1980 , comporte 12 paliers. La navigation à grand gabarit y est active depuis de nombreuses années.
Le Haut-Rhône, de Lyon à Pougny, est en cours d'aménagement : 5 paliers sont réalisés, celui de SaultBrenaz est en cours de construction, celui de Loyettes est en projet. La navigation commerciale n'y existe pas pour l'instant.

Dès son achèvement, le Rhône comportera 19 aménagements dont la puissance installée sera de $3080 \mathrm{MW}$ et la production annuelle de $16,7 \mathrm{TWh}$.

Les aménagements comportent une retenue créée par un barrage (figure 2), un canal de dérivation, une usine hydroélectrique à laquelle est accolée une écluse (pour le Bas-Rhône uniquement), un canal de restitution (2). De part et d'autre de la retenue subsistent des champs d'inondation. L'aménagement du fleuve est pratiquement continu, le remous créé par un barrage remonte à la restitution de l'aménagement amont.

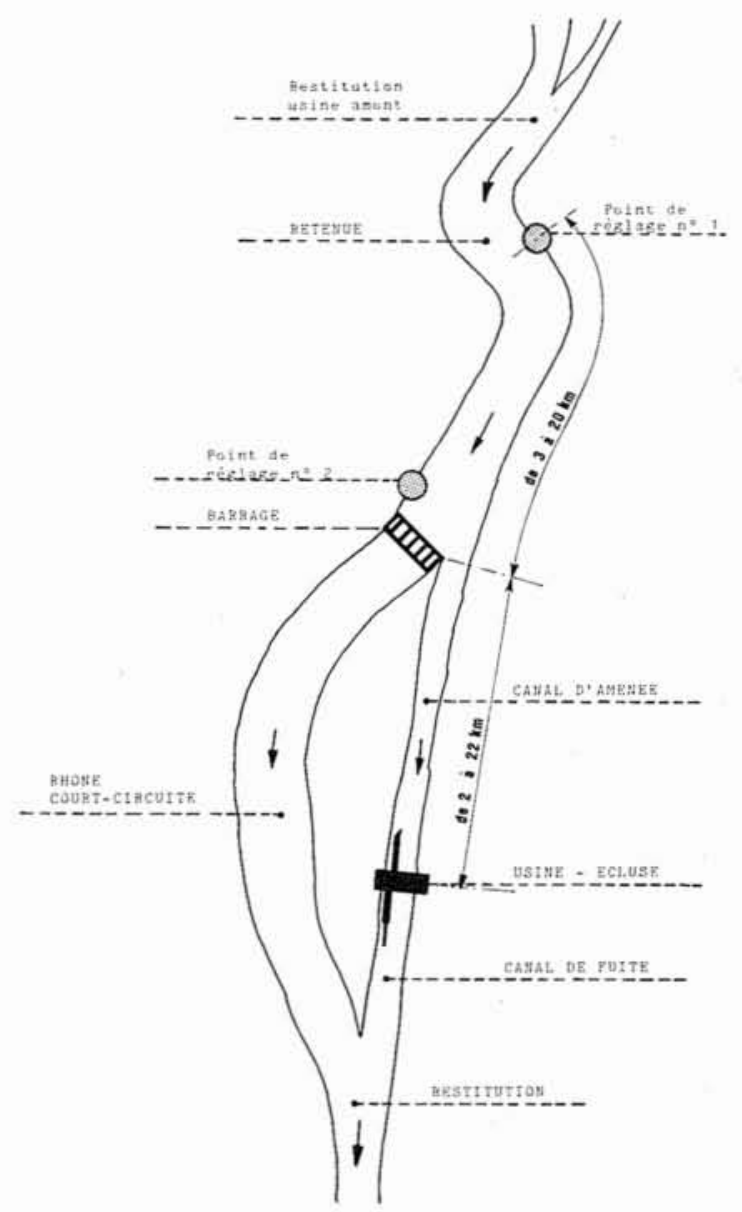

Figure 2. - L'aménagement type du Rhône.

(1) Il faut ajouter à celà, la volonté manifestée par l'exploitant puis le concepteur, depuis 1970, de supprimer les postes d'agents de conduite (les services continus) pour cause de pénibilité.

(2) Toutefois trois aménagements ne comportent pas de canal de dérivation, ce sont Génissiat et Seyssel sur le Haut-Rhône et Vaugris sur le Bas-Rhône. 


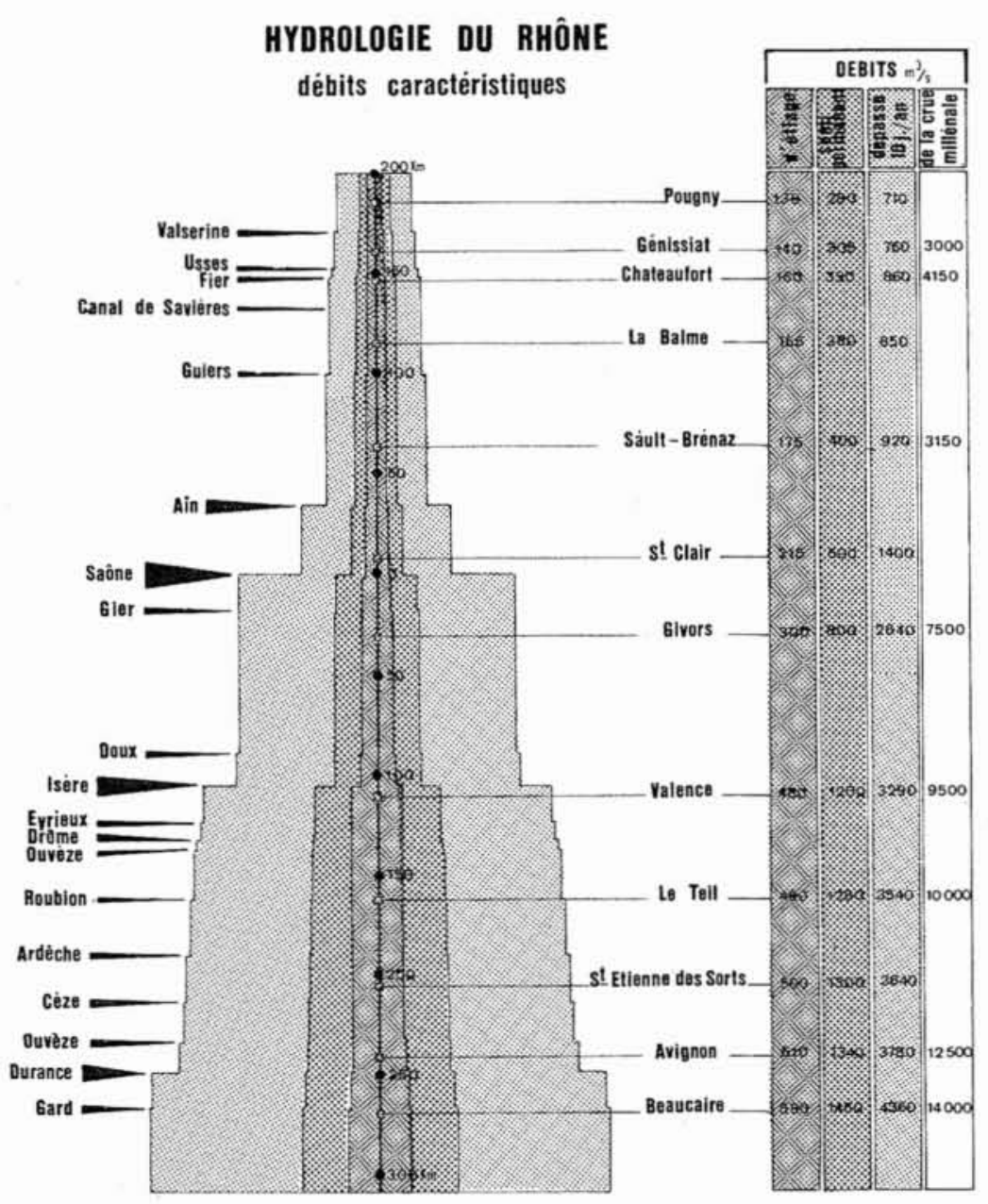

Figure 3

On dénombre :

- 25 barrages dont 22 barrages mobiles comportant en tout 193 vannes dont les largeurs varient de 15 à $45 \mathrm{~m}$ et les hauteurs de $7 \mathrm{~m}$ à $15 \mathrm{~m}$,

- 20 centrales dont 19 de basse chute, comportant 83 groupes turbine-alternateur : 6 de type Francis, 33 de type Kaplan verticale et 44 de type bulbe.

Le débit semi-permanent du Rhône passe de $320 \mathrm{~m}^{3} / \mathrm{s}$ à Génissiat à $1600 \mathrm{~m}^{3} / \mathrm{s}$ à Vallabrègues et le débit de la crue millénale est estimé à $7500 \mathrm{~m}^{3} / \mathrm{s}$ à Lyon et $14500 \mathrm{~m}^{3} / \mathrm{s}$ à Arles (figure 3).

\section{Généralités sur les automates du Rhône}

\subsection{La structure des automates}

L'automatisation des aménagements hydroélectriques du Rhône a été conçue suivant une structure hiérarchisée à trois niveaux : a) Chaque aménagement comportant centrale et barrage est conduit d'une manière totalement autonome et automatique par un calculateur industriel de conduite de processus. Il prend en compte, pour la régulation automatique du plan d'eau les caractéristiques propres à l'aménagement :

- comportement hydraulique de la retenue,

- consignes diverses d'exploitation : fonctionnement au fil de l'eau, en placement d'énergie, réglage des niveaux $\mathrm{Z}$ en fonction du débit $\mathrm{Q}$ selon des lois $\mathrm{Z} / \mathrm{Q}$ imposées par les cahiers des charges,

- le régime hydrologique du fleuve et de ses affluents,

- les caractéristiques des organes de réglage du débit : les turbines (réglables ou non réglables), les vannes de décharge, les vannes et volets des barrages, les seuils déversoirs, les digues submersibles.

b) Un poste central de contrôle et de surveillance doté également d'un (ou deux) calculateurs situé à Chateauneuf-du-Rhône, à peu près au milieu de la chaîne d'aménagements du Bas-Rhône, un poste identique, situé à Génissiat en tête de chaîne pour le Haut-Rhône. Chacun de ces postes : 
- reçoit de chaque aménagement des informations : niveaux, débits, état des installations qui lui permet d'assurer la télésurveillance de l'ensemble de la chaine,

- transmet à chaque calculateur d'aménagement, en temps réel ou en temps différé, les paramètres de fonctionnement des centrales en éclusée : modulation de débit et donc de puissance pour le placement de l'énergie.

c) Enfin, un troisième niveau est constitué, au plan de chaque aménagement, par un dispositif dit de Sécurité Intrinsèque complètement indépendant du calculateur de conduite, et qui assure, en ultime secours, la protection contre tout exhaussement et abaissement anormal de la retenue.

\subsection{L'automate de conduite}

L'automate de conduite assure trois types de fonctions :

- acquisition - traitement et surveillance de toutes les grandeurs caractéristiques de la retenue, des états et de la disponibilité de toutes les installations, ainsi que des ordres et consignes émis par l'exploitant;

- conduite du plan d'eau, c'est-à-dire calculs des débits à faire sortir de l'aménagement en fonction du régime hydrologique du fleuve, de l'état de la retenue, des consignes de niveaux ou de débit maxi et mini, des ordres reçus, des perturbations enregistrées, des contraintes imposées par les organes de réglage du débit;

- commande directe des organes réglant le débit, contrôle du bon fonctionnement de ces organes et de la bonne exécution des ordres.

La complexité de la conduite dans les différents régimes d'exploitation, dans les transitions entre ces différents régimes, l'importance primordiale accordée à la sécurité compte tenu des diverses possibilités de perturbations de toute nature (hydraulique, mécanique, électrique) conduisent à étudier avec précision le comportement hydraulique de l'aménagement et à réaliser des simulations du système pour un éventail de perturbations le plus large possible. Le comportement hydraulique est étudié sur modèle mathématique et les simulations réalisées sur ordinateur.

\subsection{Le modèle mathématique}

Chaque modèle représente un aménagement hydraulique délimité à l'amont par l'origine du canal de fuite et par l'origine du tronçon de fleuve court-circuité de l'aménagement amont, et à l'aval par la centrale et le barrage de l'aménagement concerné. Il inclue par conséquent la retenue, le canal et le cas échéant les affluents et les champs d'inondation maintenus pour l'amortissement des crues.

C'est un modèle à caractère classique qui utilise une méthode de résolution implicite des équations aux différences finies déduites des équations de Saint-Venand. Le modèle (figure 4) est découpé et représenté numériquement
mOdele mathematique DE L'amenagement de ChaUtAgne schẻma topologique simplifié

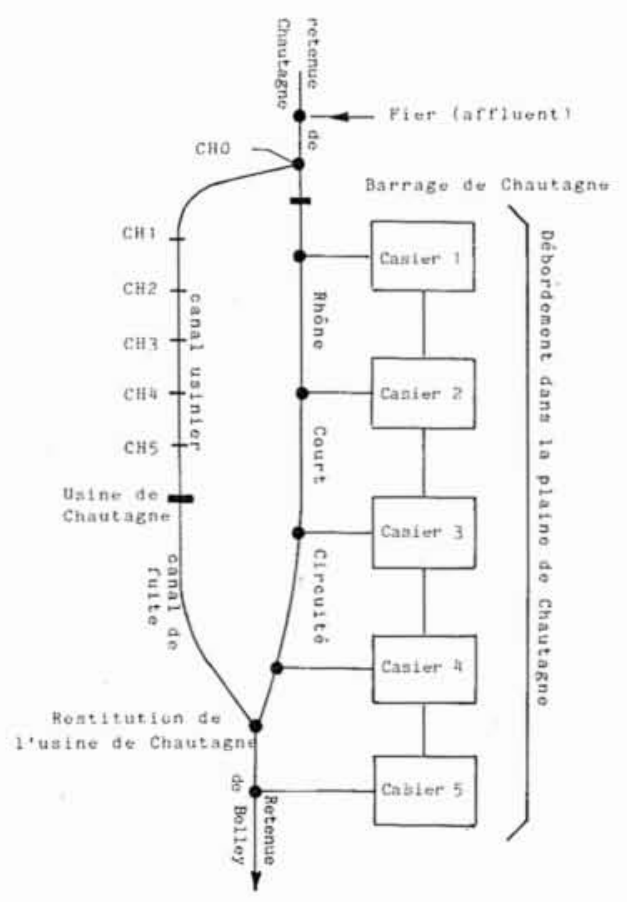

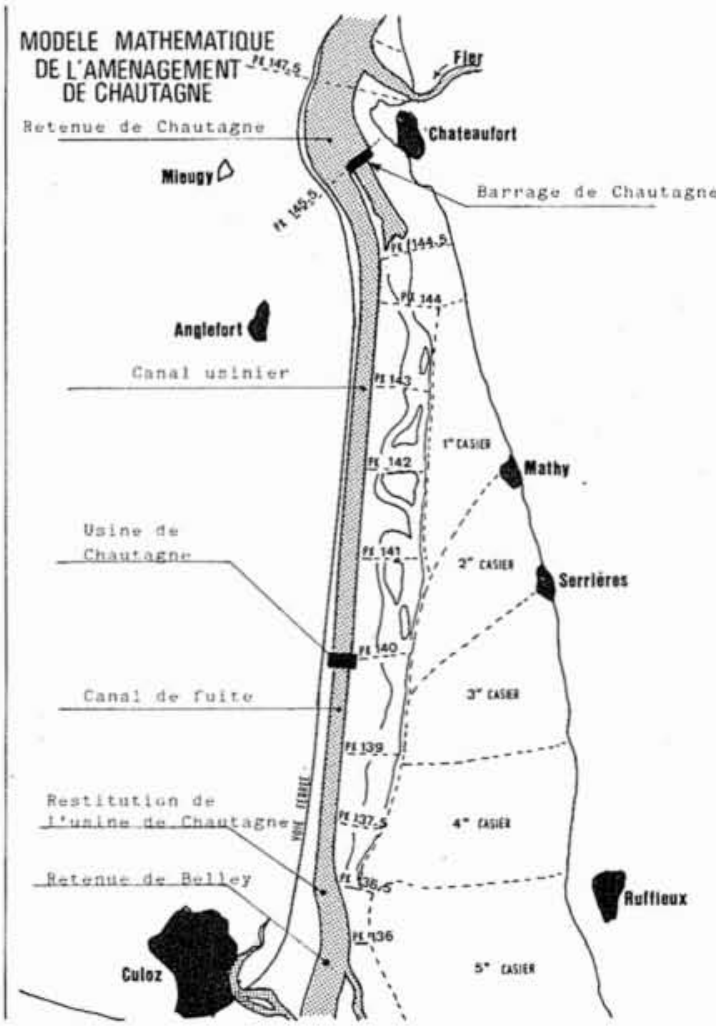

Figure 4 
MODULATION DE DEBIT fonetionnement en eclusée cumulative

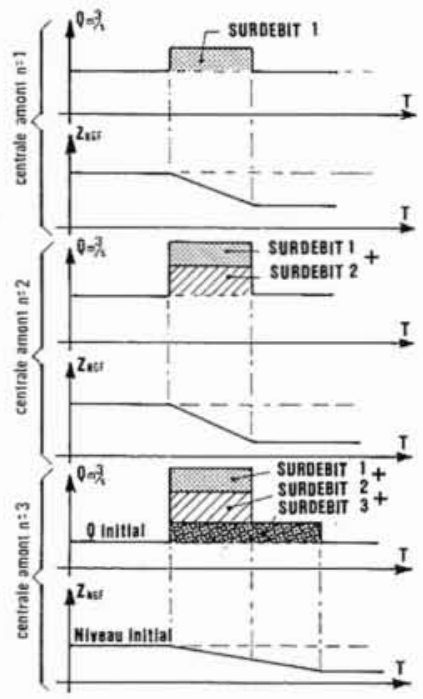

MODULATION DE DEBIT

fonctionnement à volume constant

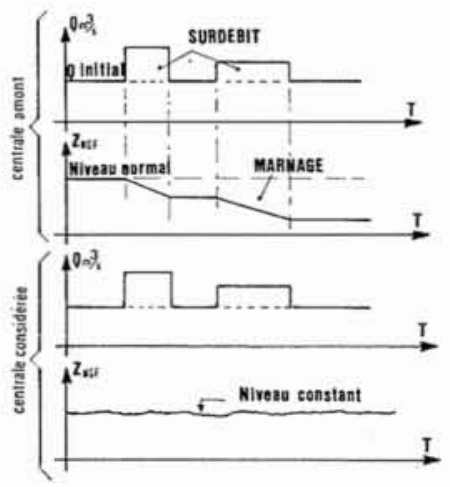

MODULATION DE DEBIT

fonctionnement en éclusée avec destockage dans la retenue
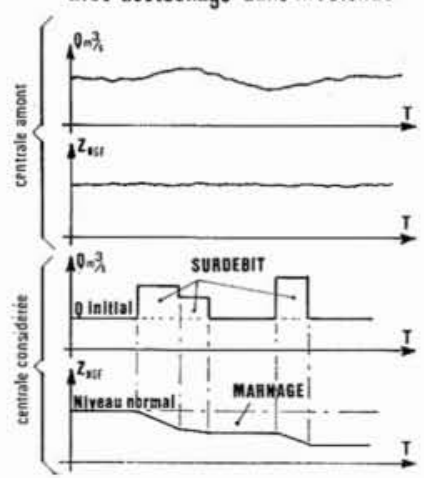

Figure 6

\section{PEAGE DE ROUSSILLON}

Consignesd'exploitation

(1) -à l'entrée du canal

(2) $\rightarrow$ à l'amont de la retenue

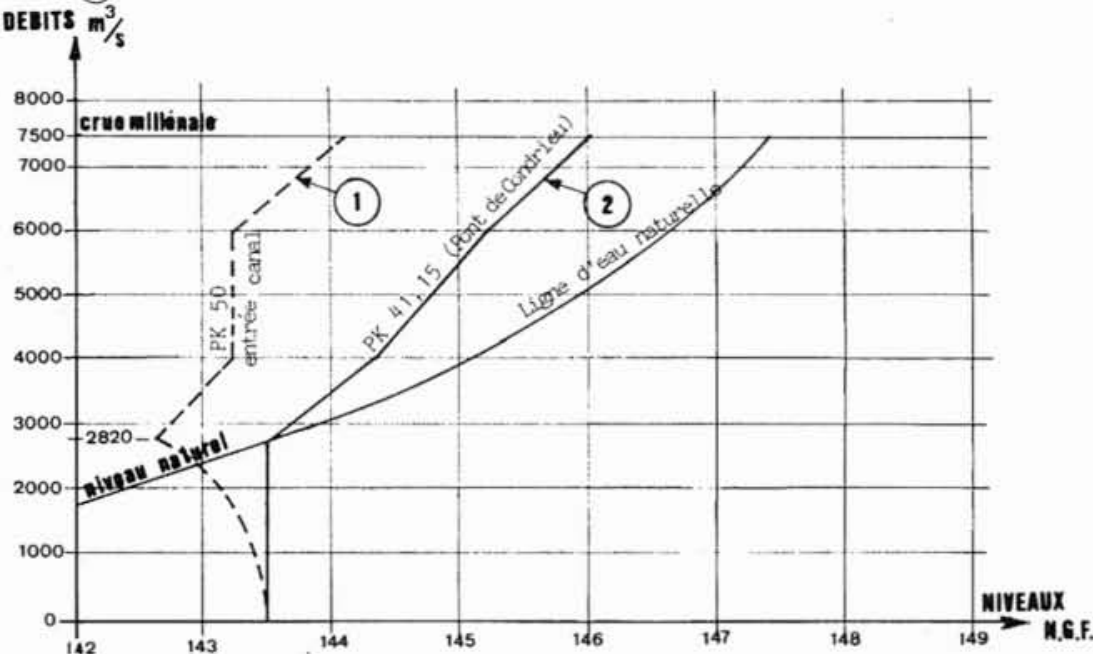

Figure 5

COMPORTEMENT DES RETENUES exemple de loi de surface et temps de réponse
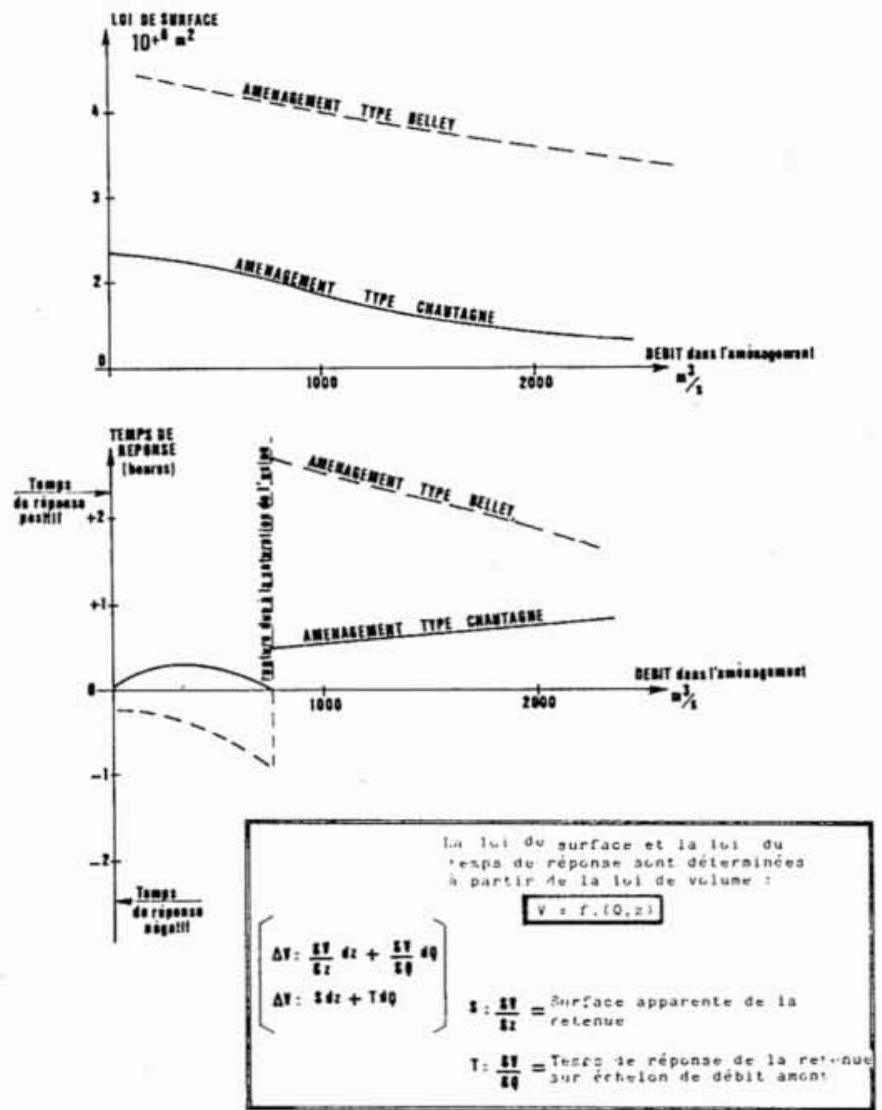

Figure 7 
de façon à simuler le plus parfaitement possible les variations de volume qui sont des éléments essentiels de la régulation, ainsi que les propagations d'onde. Le modèle est constitué à partir de profils (un par kilomètre en moyenne et par point singulier) et de lignes d'eau de projet et prend en compte toute la géométrie du lit (mineur et majeur) de la retenue, des canaux, de l'emplacement des ouvrages de réglage de débit ou d'écoulement traversier (seuils, digues submersibles, etc.) le modèle est étalonné à partir des lignes d'eau aussi bien pour les débits usuels que pour les débits de crue connus ou estimés (millénale).

\subsection{Le comportement hydraulique de la retenue}

Le comportement hydraulique de la retenue est testé, non seulement en régime permanent, mais aussi et surtout en régimes pseudo-permanents et transitoires.

En effet, sur un fleuve comme le Rhôhe, le régime d'écoulement est très peu souvent permanent, car le débit est constamment variable et les organes de réglage des divers aménagements existants à travers lesquels il passe contribuent également dans une certaine mesure à sa modulation. Aux débits usuels, même quand le régime d'exploitation est celui de "fil de l'eau", les débits horaires peuvent varier très sensiblement sur une période de 24 heures.

En crue, des gradients de 100 à $400 \mathrm{~m}^{3} / \mathrm{s}$ par heure pendant quelques heures peuvent être observés. De plus, les consignes d'exploitation (figure 5) imposent des niveaux variables en fonction du débit, ce qui entraine des phénomènes de vidange ou de remplissage des retenues. Des affluents importants peuvent débiter directement dans ces retenues influençant ainsi l'écoulement.

Quand le régime d'exploitation est celui de l'éclusée en placement d'énergie, les modulations de débit avec ou sans prélèvement d'un volume d'eau dans la retenue, qui peuvent être quotidiennes en certaines périodes de l'année, excluent tout espoir de trouver des régimes d'écoulement établis durables.

Par ailleurs, des événements inhérents au fonctionnement des centrales hydroélectriques génèrent des régimes transitoires assez sévères qu'il est nécessaire de maîtriser.

En particulier, les disjonctions, qu'elles soient dues à des défauts internes aux machines ou au réseau de transport, induisent des variations de débit à la centrale plus ou moins brutales. De plus, si la coupure de débit à la centrale est importante ou durable, il y a nécessité d'ouvrir le barrage afin d'éviter un exhaussement exagéré du niveau de la retenue. De telles manœuvres, quand elles se produisent sur l'aménagement amont, créent pour l'aménagement aval une brusque modification dans la provenance du débit entrant : ce qui arrivait par l'usine amont est partiellement ou totalement réduit, et n'est compensé qu'avec retard, par ce qui vient du barrage amont - inversement à la reprise de charge à la centrale amont, c'est un surdébit entrant qui peut être constaté à la restitution du fait de la conjonction avec le débit qui continue à s'écouler dans le Rhône court-circuité, malgré la fermeture du barrage.

Des simulations devront donc porter sur des cas de fonctionnement tels que les suivants : a) En exploitation dite " normale"

- fonctionnement en modulation de débit (figure 6)

- transfert, à volume constant, de créneaux de débit venant de l'amont,

- exécution de créneaux de débit par prélèvement puis stockage dans la retenue,

- cumulation, c'est-à-dire reconduction des créneaux venant de l'amont avec adjonction en synchronisme ou non de créneaux résultant de la participation propre de la retenue;

- fonctionnement à niveau constant, pour tous débits;

- fonctionnement à niveau évolutif prédéterminé $Z(Q)$ ou $Z(T)$.

b) en exploitation dite perturbée

Il s'agit des fonctionnement ci-avant, mais affectés par des :

- indisponibilités d'organes amont ou locales;

- disjonctions partielles ou totales, amont, locales, avec et sans transfert de débit par le barrage;

- invalidité de mesures, une ou plusieurs.

L'analyse de ces simulations conduira à la connaissance des lois de surface, de volume (figure 7), de pertes de charge, de temps de propagation, etc. L'étude de la loi de volume $V(Q, Z)$ en fonction du débit transitant dans le bief et du niveau fixé au point de consigne exprimée de la manière suivante :

$$
d V=\frac{\delta V}{\delta Q} d Q+\frac{\delta V}{\delta Z} d Z \text { ou } d V=T d Q+S d Z
$$

permet de préciser les composantes fondamentales du comportement de la retenue : $\delta V / \delta Q=T$, expression du temps de réponse de la retenue, au point de référence, à une variation de débit; $\delta V / \delta Z=S$, expression de la surface apparente de la retenue au point de référence.

Des valeurs de $T$ vont dépendre les performances et qualités demandées à la régulation. Des valeurs nettement positives laisseront présager des difficultés pour maîtriser les conséquences des variations de débit locales par exemple sur disjonctions (figure 8) tandis que des valeurs très négatives entraineront des difficultés pour maîtriser les variations de niveaux découlant des variations de débit venant de l'amont ${ }^{(3)}$.

Si $T$ est voisin de zéro, les deux types de perturbations présenteront des contraintes comparables.

De même, de l'importance et de la loi d'évolution de la surface apparente dépendra la sensibilité de la retenue aux écarts entre débit entrant et débit sortant.

(3) Par exemple, pour un point de réglage situé loin en amont dans une retenue, toute variation de débit entrant aura des répercussions à très court terme sur le niveau en ce point, niveau qui ne pourra être maitrisé et maintenu aux alentours de sa valeur de consigne que si la manceuvre en débit a été convenablement évaluée et anticipée par l'ouvrage aval. Hors, la prévision des variations du débit entrant n'est pas toujours possible - sauf dans le cas de marche sur programme $Q(t)$ - d'où la difficulté de régler les niveaux dans un tel aménagement à temps de réponse négatif. 


\section{DECLEMCHEMEWT TOTAL SUR UME RETEMUE DE FAIBLE SURFACE AVEC TEMPSS DE REPONSE "POSITIF" \\ DECLENCHEMEMT TOTAL SUR UME RETEMUE MOYEMNE AVEC TEMPS DE REPOHSE "NETTEMENT MEGATIF"}
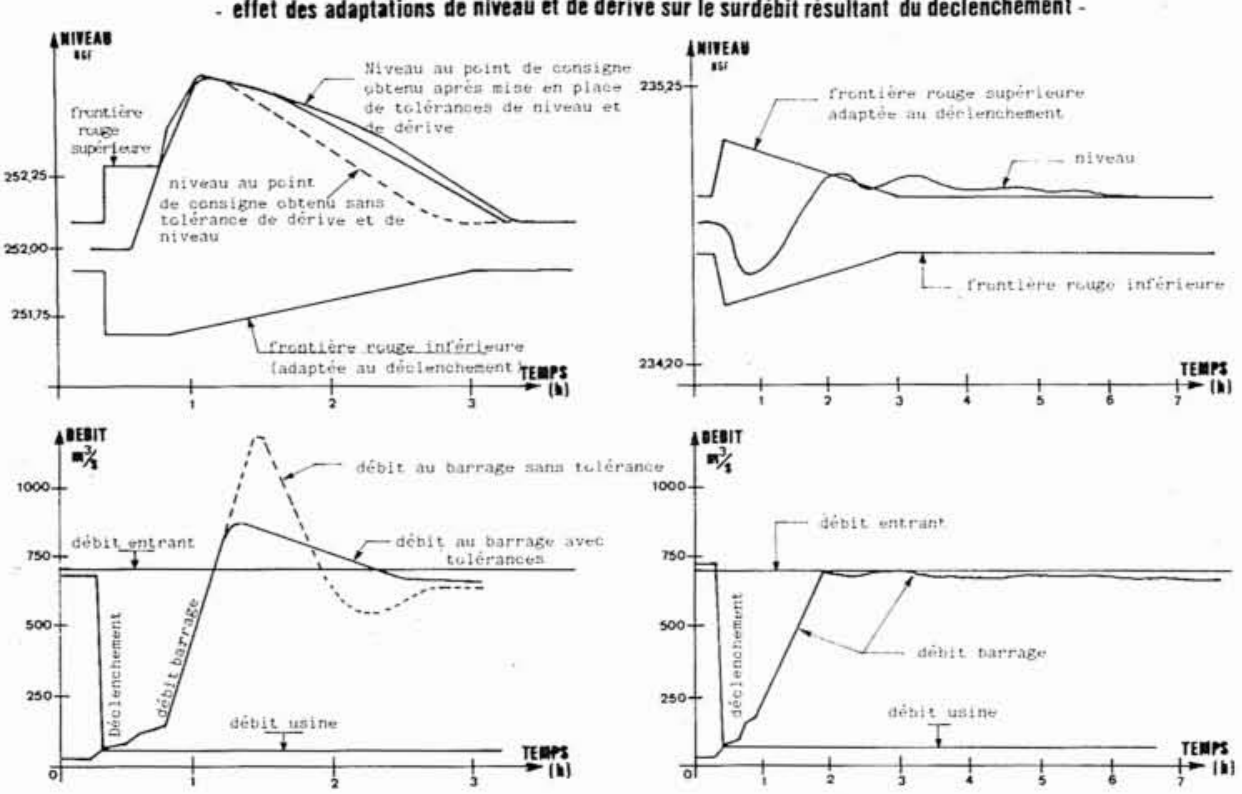

Figure 8

\section{PRINCIPE DES ECLUSEES CUMULATIVES}
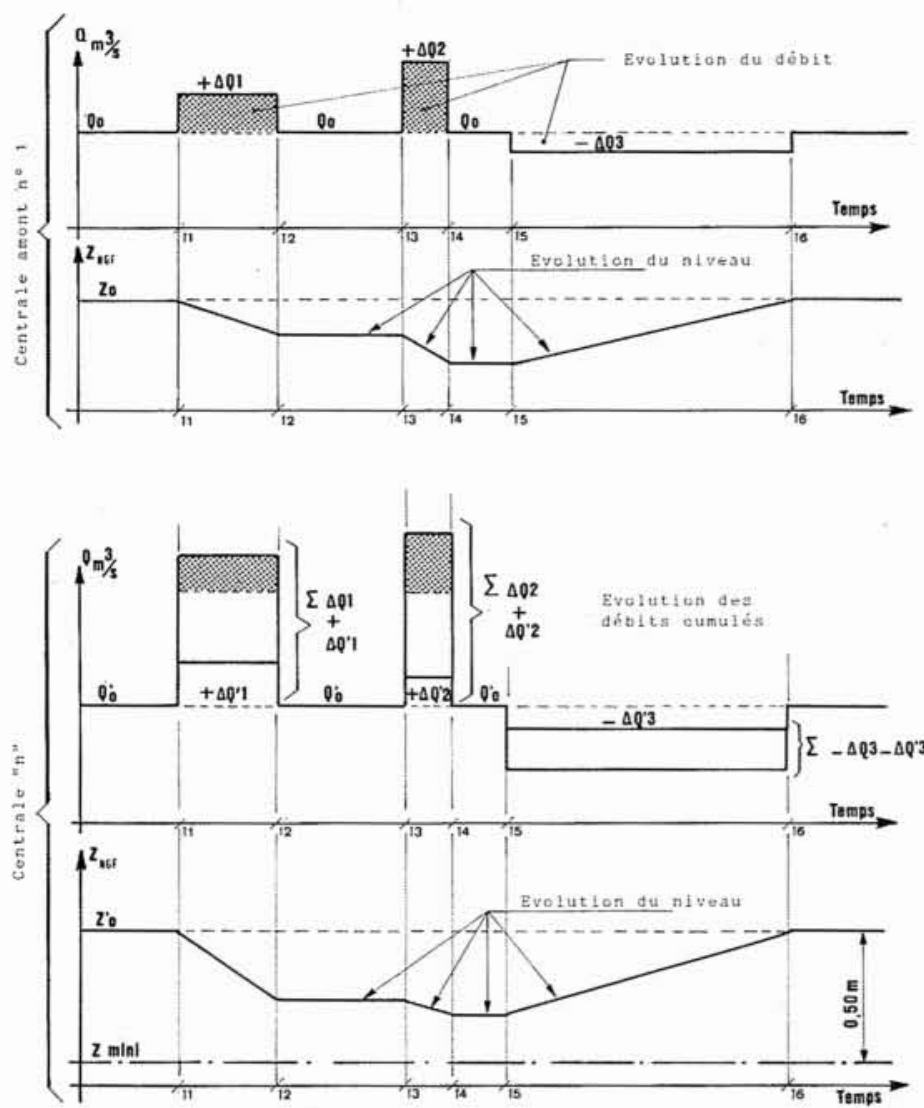

Figure 9
Sur la figure 8 sont donnés deux exemples illustrant : - les variations de niveau au point de contrôle qui résultent d'une disjonction (coupure rapide d'un débit de $700 \mathrm{~m}^{3} / \mathrm{s}$ à la centrale) dans deux retenues: Chautagne à temps de réponse négatif et Belley à temps de réponse positif;

- Les débits à évacuer par le barrage calculés par la régulation afin de rétablir le niveau initial de la retenue : en trait tireté avec une loi de régulation habituelle, en trait plein après adaptation de cette régulation par adjonction de tolérances sur la consigne supérieure de niveau (frontière rouge).

L'étude des réactions de la retenue en régime permanent, pseudo permanent et transitoire, et la connaissance précise des différentes lois citées plus haut permettra de définir les moyens à mettre à la disposition de la régulation : nombre et emplacements des points de mesure de niveaux et de débits redondance des informations nécessaires, etc., fonctions à assurer - algorithmes banalisables ou spécifiques. A ce stade de l'étude, on dispose de tous les éléments pour entreprendre la construction des modules de régulation et de conduite numérique du fleuve.

\subsection{L'automate de régulation}

La fonction de cet automate est la détermination d'un débit objectif à sortir de l'aménagement respectant les objectifs de production d'énergie, de valorisation ou de placement de cette énergie, de sécurité, de navigation (pour le Bas-Rhône).

L'objectif de production d'énergie dépend du débit du fleuve, de la demande formulée par le dispatching. Il est variable ou modifiable à tout instant. 


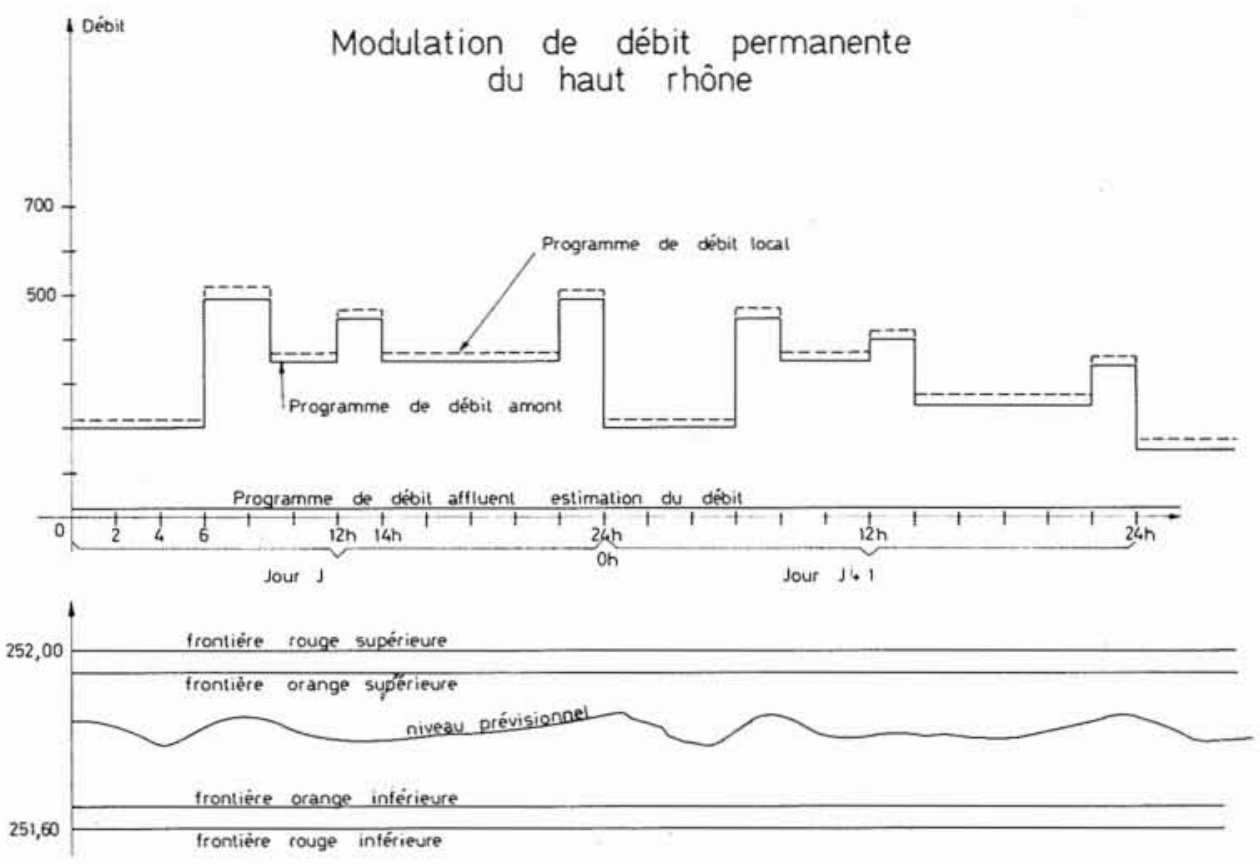

Figure 10

a) Au fil de l'eau, on cherchera à optimiser la relation production-débit, en exploitant la retenue à la cote maximale permise par le cahier des charges. Le fonctionnement consiste alors en un réglage de niveau et asservissement à une consigne qui peut être constante ou variable suivant le débit du fleuve et suivant les aménagements.

b) En éclusée conventionnelle, type Bas-Rhône (figure 9), la production est modulée à la demande du dispatching. La surpuissance réalisée est obtenue par marnage de la retenue. Le fonctionnement consiste alors à faire un réglage en débit suivant un programme $Q(T)$. La surpuissance réalisée, la retenue est alors exploitée à niveau bas, au fil de l'eau. Le réglage est alors un réglage en niveau sur une consigne basse. A la période de faible demande d'énergie, on fait correspondre une période de rétention : le volume d'eau destocké est reconstitué, le niveau est remonté à sa valeur de consigne haute. Le réglage consiste alors à faire une visée de niveau: un niveau prédéterminé est réalisé à l'échéance d'un temps donné.

$\mathrm{Ce}$ fonctionnement en éclusée peut conduire à des surdébits importants, par exemple plus de $500 \mathrm{~m}^{3} / \mathrm{s}$ sur un débit support de $1000 \mathrm{~m}^{3} / \mathrm{s}$, d'où basculements de plans d'eau importants répétés plusieurs fois par jour;

c) En pointe : ce fonctionnement correspond à un appel brutal de puissance pour soutenir un réseau défaillant dans l'attente de démarrage d'un autre moyen; l'urgence est telle que la prise de charge se fait à une vitesse maximale 4 à 5 fois supérieure à la vitesse habituelle. Un certain nombre de centrales sont poussées brutalement à saturation, ce qui entraine des variations de débit de très grande amplitude effectuées à grande vitesse et sollicitant tous les organes de réglage de débit ${ }^{(4)}$.
Le fonctionnement en éclusée ou en pointe doit cependant rester compatible avec la navigation commerciale qui ne doit pas être gênée, d'où découlent des objectifs contraires: les niveaux et les débits ne doivent pas descendre en dessous de seuils minimaux (maintien d'un mouillage minimum garanti).

d) En modulation de débit permanente (type HautRhône) (figure 10)

Ce type d'exploitation est différent de celui des éclusées conventionnelles, en ce sens que la modulation en débit est permanente. Il s'agit donc d'un fonctionnement sur programme de débit $Q(T)$ renouvelé de jour en jour sans qu'il y ait interruption de programme. Ce fonctionnement se perpétue tant que le niveau, de ce fait essentiellement variable, se maintient dans le domaine qui lui est assigné. Si le niveau sort de ce domaine, une régulation de niveau est mise en œuvre pour le stabiliser sur une cote seuil, puis un guidage ramène ce niveau dans le domaine assigné. A ce moment, le réglage en débit reprend.

e) En téléréglage, le fonctionnement consiste à participer à la modulation de puissance, aléatoire en amplitude en fonction de la variation de fréquence du réseau électrique (figure 11).

La participation au téléréglage se traduit par des modulations de débit atteignant 5 à $10 \%$ du débit moyen actuel, modulations dont les variations d'amplitude aléatoires constituent des perturbations parasites permanentes.

(4) La durée de cette surpuissance est fixée en raison inverse de son amplitude; le marnage autorisé dans les retenues étant limité, le volume d'eau de stockage est lui aussi limité. 


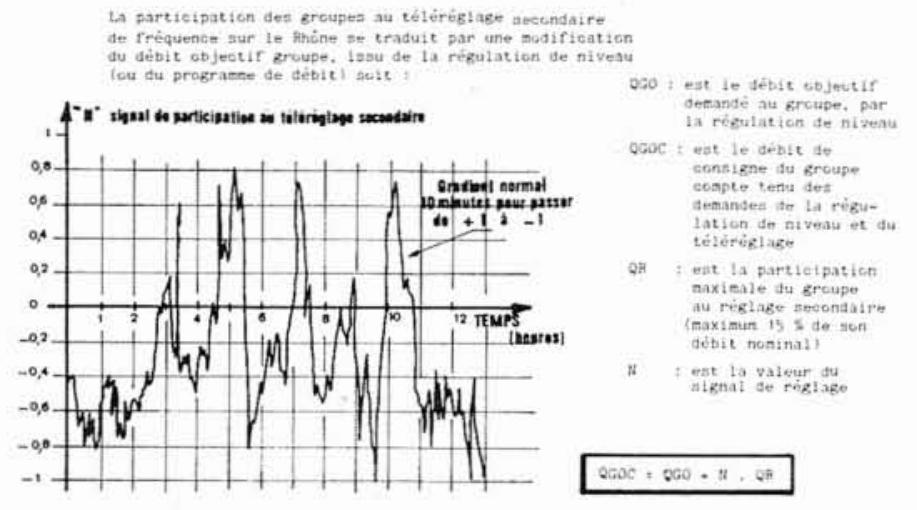

Figure 11. - Téléréglage secondaire de fréquence des usines eu fil de Teau.

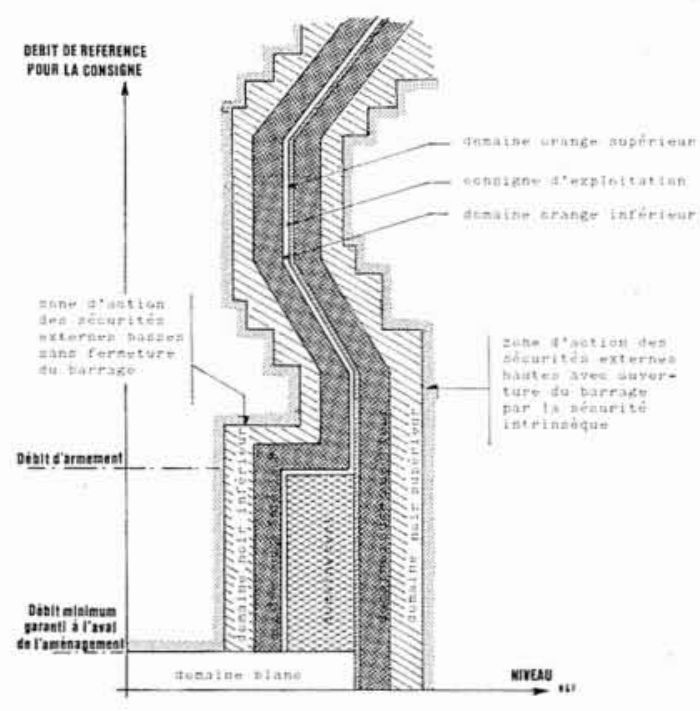

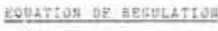

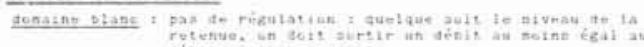

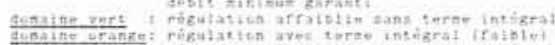

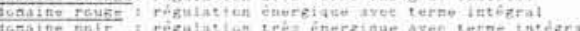

Figure 13. - Domaines de niveaux et de débits.

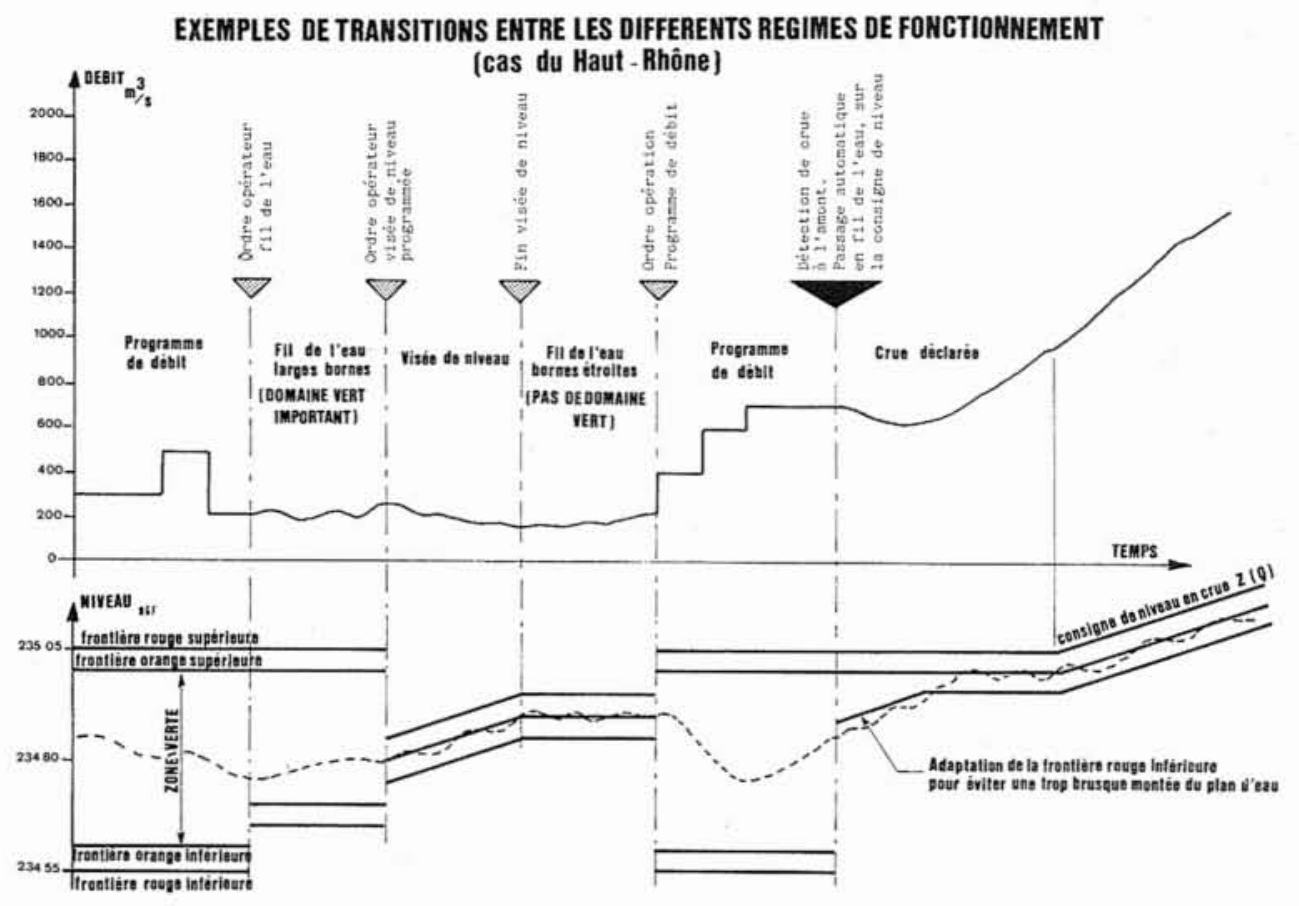

Figure 12 
Il est donc nécessaire, pour atténuer les effets de ces perturbations sur la tenue du plan d'eau, de désensibiliser la régulation de niveau et de donner à ce dernier un minimum de liberté d'évolution. tés :

Les objectifs de sécurité multiples doivent être respec-

- la protection contre les inondations limite la cote de la retenue à une consigne supérieure variable avec le débit. L'effet cumulatif de la chaîne d'aménagements implique la non amplification des débits et des vitesses moyennes de variation de débit, la non accélération de la propagation des débits le long du fleuve;

- la navigation et les riverains peuvent être gênés par l'amplitude et la fréquence des manœuvres : il faut donc éviter les manœuvres inutiles, brutales ou hors normes;

- la protection ultime des ouvrages étant assurée par des sécurités externes, indépendantes de l'automate principal, il est nécessaire de prévenir leur excitation, même en cas d'accident de débit (déclenchement par exemple);

- la suppression du personnel de quart nécessite une grande sécurité de fonctionnement afin qu'après un incident, le personnel puisse se consacrer à la réparation de celui-ci. L'automate doit donc conserver un fonctionnement sûr, même en cas de défauts multiples;

- la conduite automatique doit être aussi sûre quel que soit le débit du fleuve, du débit d'étiage au débit de la crue centenale, quelle que soit la brutalité des apports provoqués par des affluents capricieux;

- le personnel astreint à domicile doit recevoir des alarmes hiérarchisées et circonstanciées.

L'automate de régulation devra en conséquence comporter un certain nombre de modules fonctionnels dont l'organisation et la structure seront organisées autant que faire se peut de manière à ce que la plus grande partie d'entre eux soient portables, c'est-à-dire banalisables (aux paramètres près) d'un aménagement à l'autre, et les autres spécifiques pour tenir compte des particularités de chaque aménagement.

Avant d'implanter ces modules dans les calculateurs de conduite d'aménagement, il est indispensable de procéder sur le modèle à de très nombreuses simulations en boucle fermée pour tester et régler les différents algorithmes et paramètres des modules de régulation. Ceux-ci sont d'abord testés dans les conditions normales d'exploitation, puis la mise au point faite, les tests se poursuivent dans les conditions d'exploitation dégradées.

Une très grande attention doit être portée dans l'analyse des transitions qui constituent une des difficultés à vaincre (figure 12), ces difficultés ne découlent pas des situations usuelles stables mais des perturbations et événements exceptionnels.

\section{La régulation (cas du Haut-Rhône)}

\subsection{Généralités}

La méthode de détermination du débit objectif à sortir de l'aménagement est différente suivant le mode d'exploitation. a) Au fil de l'eau, le débit est issu d'une boucle de régulation de niveau.

b) En modulation de débit pour le placement d’énergie, le débit est issu :

- d'un programme de débit $Q(T)$ tant que le niveau de la retenue reste dans un domaine assigné;

- d'une boucle de régulation de niveau, si le niveau de la retenue à tendance à sortir du domaine assigné.

Le débit évolue entre deux extrêmes:

- un débit minimum garanti à sortir de l'aménagement, en dessous duquel les régulations en niveau et en programme de débit ne sont plus prioritaires. L'objectif est de sortir au moins ce débit minimum;

- un débit égal au débit d'équipement de la centrale, au-delà duquel le mode d'exploitation fil de l'eau est imposé.

Dans cet intervalle, les deux types de réglage (niveaux ou débits) sont possibles.

On distingue cinq domaines de niveaux définis par rapport à la cote normale d'exploitation (cahier des charges) (figure 13).

- une zone verte dans laquelle le niveau de la retenue peut évoluer librement pendant l'exécution du programme de débit $Q(T)$;

- deux zones orange, haute et basse, dans lesquelles il peut $y$ avoir action de correction sur le niveau, pendant l'exécution du programme de débit, si ce niveau, par suite de circonstances particulières, venait à y pénétrer;

- deux zones rouge, haute et basse, dans lesquelles le réglage en niveau prend le pas, le niveau de la retenue ne pouvant, en aucun cas, dépasser un seuil haut ou un seuil bas.

\subsection{La régulation de niveau}

On peut noter ici que la régulation des retenues étant basée essentiellement sur la connaissance de l'évolution des niveaux en des points situés loin en amont (de 5 à $20 \mathrm{~km}$ suivant les aménagements) des organes de réglage de débit et sur la connaissance de l'évolution des débits entrants (aménagements amont et affluents), il est nécessaire de disposer de télétransmissions très fiables.

La régulation de niveau doit tenir compte :

- des fluctuations rapides qui peuvent être temporairement importantes (vagues de vent ou batillage);

- des variations lentes représentatives des volumes d'eau dans la retenue;

- des ondes transitoires dont l'effet peut être notable pendant plusieurs heures (ondes de disjonction par exemple).

Seules les variations lentes peuvent être contrôlées par une action judicieuse sur les débits sortant de l'aménagement. Les fluctuations rapides doivent être éliminées par un filtrage adéquat des mesures. Par contre, on ne peut pas agir sur les ondes transitoires qui masquent le phénomène important, la variation de volume de la retenue. Il faut donc s'affranchir au mieux des basculements transitoires.

La régulation de niveau est de type classique P.I.D. "proportionnel intégral dérivé ", avec entrées d'anticipation; d'autres variantes peuvent en outre être utilisées 
Figure 14. - Programme de débit Niveau prévisionnel Intervention de la régulation
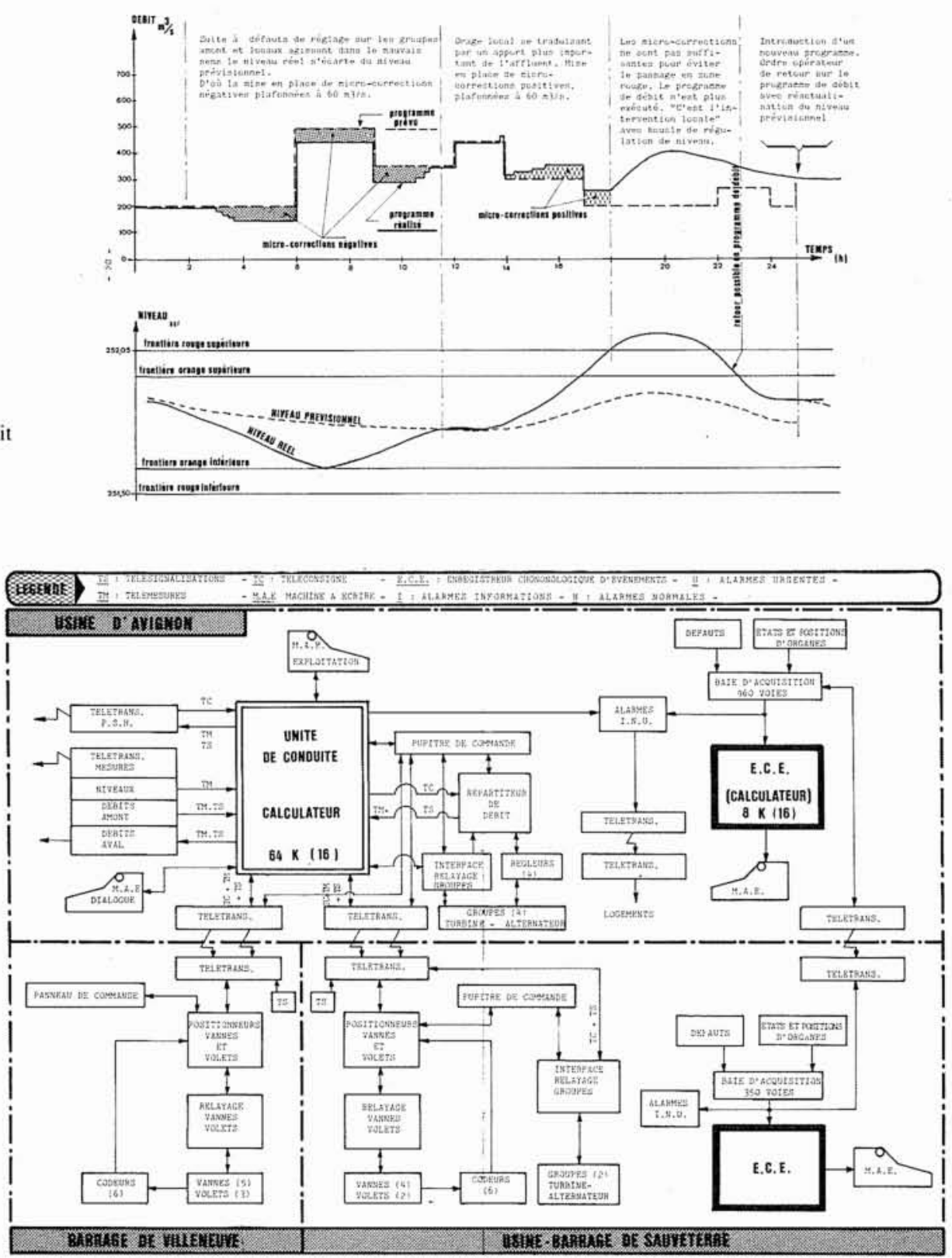

Figure 15. - Configuration des matériels d'automatisme.

suivant les objectifs à atteindre. L'équation de régulation est écrite sous forme incrémentale, sur un pas de temps de $5 \mathrm{mn}$.

Si $Q O$, est le débit objectif au pas de temps actuel, $Q O_{t-\Delta t}$ le débit objectif au pas précédent, $D Q O_{l}$ l'incrément de régulation,

la formulation est la suivante :

$$
Q O_{t}=Q O_{t-\Delta t}+D Q O_{t}
$$

L'incrément $D Q O$ se présente lui-même sous la forme générale suivante :

$$
D Q O=A \cdot D Q E+B \cdot D Z+C \cdot E Z C
$$

$D Q E, D Z$ représentent respectivement la variation, au cours du pas de temps, du débit entrant et du niveau réel de la retenue au point de réglage.

$E Z C$ représente l'écart entre le niveau réel de la retenue au point de réglage et le niveau de consigne imposé en ce point.

Les coefficients $A, B, C$ sont déterminés de façon à trouver un compromis acceptable entre les contraintes suivantes :

- avoir une insensibilité aussi grande que possible aux ondes transitoires;

- avoir un temps de réponse court;

- ne pas générer des variations de débit exagérées;

- avoir un système stable.

Ce compromis est étudié par de nombreuses simulations sur le modèle. Il doit en particulier aboutir à ce que la tenue trop stricte d'un niveau ne conduise pas à des 
variations de débit inacceptables par la régulation de l'aménagement aval.

\subsection{La régulation sur fonctionnement en programme de débit}

- Tant que le niveau reste dans le domaine vert et orange, il n'y a pas d'action de régulation. Le débit de consigne exécuté par l'automate est celui issu du programme $Q(T)$ communiqué par l'exploitant. Cependant, pendant l'exécution d'un tel programme, l'évolution du niveau réel est comparé au niveau prévisionnel (figure 14) (ce dernier est simulé par le calculateur pour les six heures à venir à partir des programmes de débit entrant et sortant qu'il a en mémoire) et si des écarts trop importants sont constatés, des micro-corrections de débit sont apportées au programme $Q(T)$. Les écarts peuvent être dus à des raisons diverses (programme de débit $Q(T)$ mal défini, évolution imprévue du débit d'un affluent, réalisation imparfaite du débit commandé, etc.).

- Si malgré la correction ci-avant, le niveau entre malgré tout en zone rouge, une action énergique de type P.I.D. est effectuée sur ce niveau de manière à stopper son évolution anormale (figure 14), puis ce niveau étant stabilisé, une action sous forme de guidage (P.I.D. sur niveau de consigne évolutif) le ramène en zone verte. Il est évident qu'au cours de cette action correctrice, l'exécution du programme de débit $Q(T)$ est quelque peu affectée, elle reprend son cours normal quant à nouveau le niveau est en zone verte.

\subsection{L'intégration de l'automate de régulation dans le système de conduite}

Quand l'automate de régulation est bien au point, ce qui a été vérifié par simulations nombreuses sur le modèle mathématique, les algorithmes fonctionnels correspondants à cet automate, c'est-à-dire les logiciels sont intégrés à l'automate de conduite et de commande, par l'intermédiaire des interfaces que sont :

- les organes de saisie de l'état de l'aménagement à savoir :

- les capteurs de niveaux et de débits, leur transmission, leurs indicateurs d'état ou de disponibilités,

- les capteurs de position d'organes de réglage du débit (turbines, vannes, etc.);

- les organes de commande des répartiteurs de débit;

- les organes d'exploitation et de dialogue homme-calculateur, à savoir les platines d'exploitation à partir desquelles sont émises les ordres et consignes opérateur.

Cette intégration qui est faite par des équipes différentes de celles qui ont étudié la régulation donne lieu, dès son achèvement, à une nouvelle simulation en plate-forme. On s'assure que les modules de régulation associés à tous les autres modules (d'acquisition, de traitement, de commande, de répartition, etc.) créés par ailleurs, " tournent " correctement par introduction d'un modèle mathématique simplifié qui permet une simulation en boucle fermée de la totalité des programmes du calculateur.

Enfin, les installations à piloter (turbines, vannes, niveaux de retenue, débits) reproduites sur maquettes simplifiées sont raccordées au système. Le calculateur de conduite dans sa configuration matérielle et logicielle (fig. 15) définitive est alors essayé en plate-forme. Tous les cas de figure et toutes les situations imaginées au moment de l'analyse du procédé sont alors essayés et des ultimes corrections apportées le cas échéant.

\subsection{L'installation sur le site}

La dernière phase est l'installation sur le site. A la mise en service provisoire, des essais réels très complets sont effectués. La conduite automatique n'est admise, mais avec surveillance humaine permanente, que lorsque tous les essais de sécurité ont été concluants. Quand la plupart des configurations réelles ont été testées, le système est mis en exploitation provisoire.

Seul un examen systématique des résultats d'exploitation permet d'affiner le réglage des algorithmes et ce contrôle peut exiger un certain temps. Certaines situations, en particulier celles se rapportant aux crues, ne pourront être totalement contrôlées tant qu'une crue importante n'aura pas eu lieu.

\section{Conclusion}

L'étude des systèmes de conduite intégrale d'aménagements hydrauliques de basse chute passe obligatoirement par la modélisation des aménagements, d'autant plus nécessaire que l'étude du comportement hydraulique de la retenue, des canaux, des champs d'inondation peut débuter alors que l'aménagement n'existe pas encore ou est en cours de réalisation. Le modèle mathématique est un outil très commode qui, par la possibilité de simulation des cas complexes et de perturbations diverses qu'il donne, permet une bonne prédétermination, avant l'arrivée sur le site, de tous les paramètres et procédures nécessaires à la conduite automatique du procédé et réduit non seulement les tâtonnements sur le site, mais le délai de mise en service industriel du système de conduite temps réel.

Les premiers systèmes de ce type ont été mis en œuvre à Vallabrègues par la C.N.R. et à Seyssel par E.D.F. en 1971, la décision d'automatiser la conduite des aménagements hydroélectriques du Rhône ayant été officialisée l'année d'avant, c'est-à-dire début 1970 .

En quinze ans, 17 automates de conduite (figure 16) ont été mis en service auxquels il y a lieu d'ajouter ceux du Poste de Surveillance Hydraulique (PSH) de Chateauneuf-du-Rhône et du Poste de Contrôle Centralisé (PCC) de Génissiat. Les aménagements postérieurs à 1970 ont été conçus dès le départ pour être conduits sans personnel de quart; ceux antérieurs à cette date ont été modernisés et automatisés après coup. Une collaboration étroite entre E.D.F. et C.N.R. s'est instaurée dès le début pour la conception, le développement et la mise en œuvre de ces automates dont on peut dire, avec une décennie de recul, qu'ils ont rempli le rôle industriel qui leur était assigné, et assuré dans des conditions de sécurité satisfaisantes la gestion automatique du fleuve en période de débits usuels, comme en période de crue. 


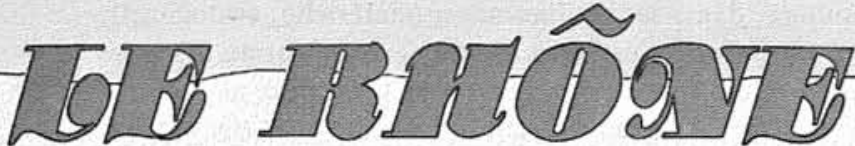

CONDUITE AUTOMATIQUE DES AMENAGEMENTS hYDROELECTRIQ̣UES ET RESEAU DE TRANSMISSION

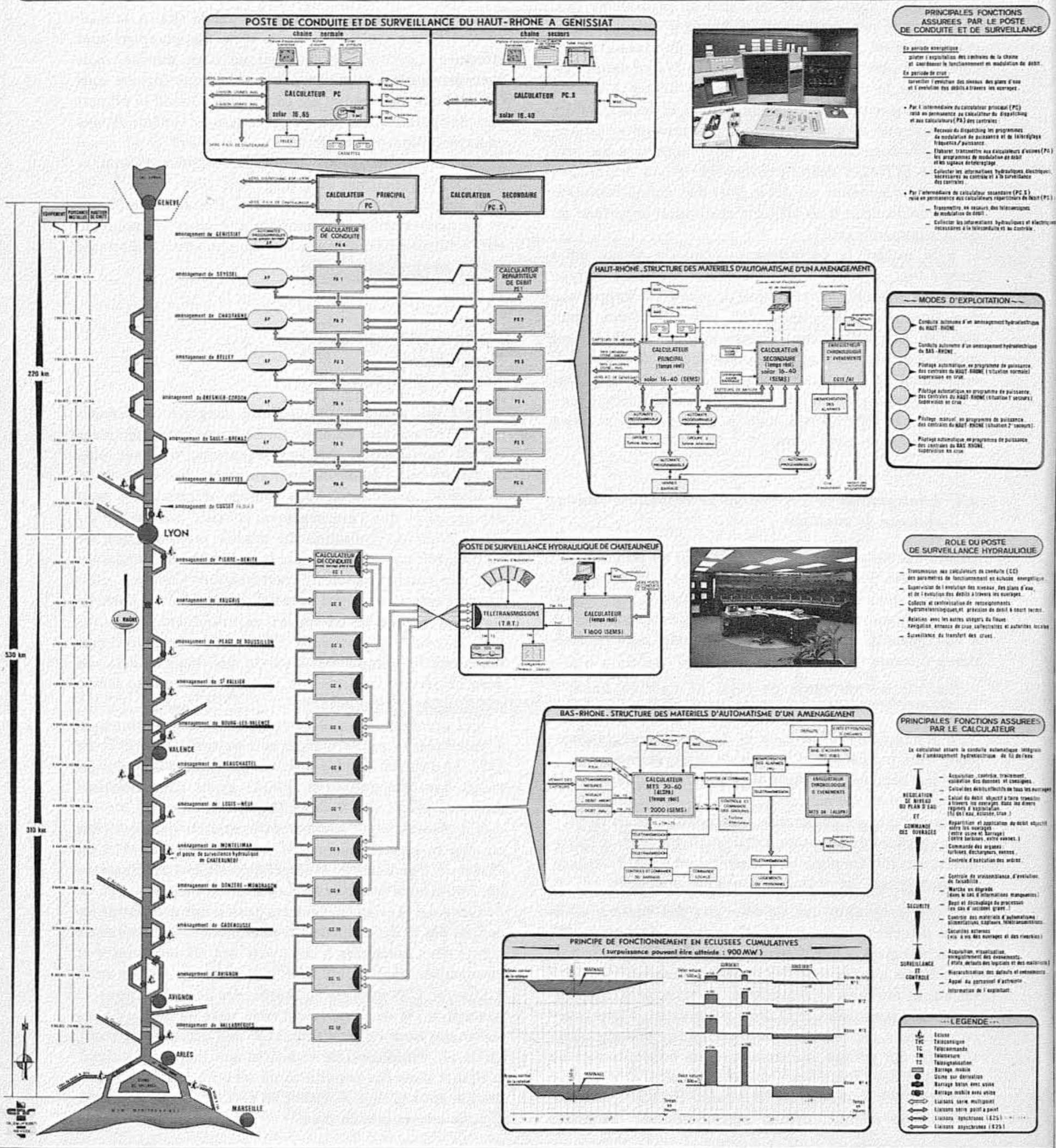

